\title{
Distributed generation and demand response dispatch for a virtual power player energy and reserve provision
}

\author{
Pedro Faria, Tiago Soares, Zita Vale, Hugo Morais
}

\begin{abstract}
Recent changes in the operation and planning of power systems have been motivated by the introduction of Distributed Generation (DG) and Demand Response (DR) in the competitive electricity markets' environment, with deep concerns at the efficiency level. In this context, grid operators, market operators, utilities and consumers must adopt strategies and methods to take full advantage of demand response and distributed generation. This requires that all the involved players consider all the market opportu- nities, as the case of energy and reserve components of electricity markets.

The present paper proposes a methodology which considers the joint dispatch of demand response and distributed generation in the context of a distribution network operated by a virtual power player. The resources' participation can be performed in both energy and reserve contexts. This methodology contemplates the probability of actually using the reserve and the distribution network constraints. Its application is illustrated in this paper using a 32-bus distribution network with 66 DG units and 218 consumers classified into 6 types of consumer.
\end{abstract}

Keywords: Ancillary services, Demand response, Distributed generation, Electricity markets, System reserves, Virtual power players

\section{Introduction}

The specific electrical energy characteristics have led to difficulties in adapting the rules and methods used in other commodities markets to the electricity markets, causing some problems namely in the achievement of lower electricity prices [1e3].

Demand response (DR) gained increasing importance in the context of electricity markets, representing an opportunity for consumers and bringing several advantages for the whole system [4e6]. It includes all the intentional electricity consumption pattern modifications by end-use customers that are intended to change the timing, level of instantaneous demand, or the total electricity consumption [7] in response to the changes in the electricity price over time.

In the same way, yet with specific concerns and policies on environmental issues, the Distributed Generation (DG) integration in power systems has been done mainly at the level of distribution networks, with small-size units that are scattered geographically mostly based on renewable energy sources [6]. As DR and DG are distributed small-size resources, new entities responsible for the aggregation of these resources are needed in order to reach full advantages for the participants (DG owners and consumers participating in DR programs) and for the power system and electricity market as a whole.

In fact, in the traditional operation of power systems, at the distribution level, the distribution network operators should manage their network ensuring the power supply to the consumers connected to it. With the implementation of electricity markets aside with the increasing levels of existing Distributed Generation (DG) and Demand Response (DR), additional challenges have appeared in order to integrate these resources in such competitive environments. As a matter of fact, the participation of small-size resources in the electricity markets implies the existence of aggregators due to the minimum size usually required for each resource' participation. As an example, the bids submitted to electricity markets must complain to specific rules, like the minimum power offered. Usually, the electricity markets impose the minimum power installed capacity (in Mibel the producers must have power capacity higher than $1 \mathrm{MW}$ [8]) or the minimum energy volume for the bids (in Elbas the minimum energy value for producers bids is $0.1 \mathrm{MWh}$ [9]). Small-size resources are not able to comply with these rules, so aggregation is required for their participation in the market. Additionally, the small-size resources usually do not have enough skills and tools to define an adequate 
Nomenclature

$$
\begin{array}{ll}
\text { Indexes } & \\
i, j & \text { node index } \\
b & \text { bus index } \\
c & \text { consumer index } \\
g & \text { generation index } \\
\mathrm{sp} & \text { supplier index } \\
t & \text { period index }
\end{array}
$$

\section{Parameter}

9 voltage angle $\left.L^{\circ}\right]$

$B \quad$ Susceptance [S]

$\mathrm{Ca}$ fixed component of cost function [m.u./h]

$\mathrm{Cb}$ linear component of cost function [m.u./kWh]

$\mathrm{Cc}$ quadratic component of cost function $\left[\mathrm{m} . \mathrm{u} . / \mathrm{kWh}^{2}\right]$

G conductance [S]

$N_{\mathrm{B}} \quad$ total number of buses

$N_{\mathrm{C}} \quad$ total number of loads

$N_{g} \quad$ total number of generators

$N_{\mathrm{sp}} \quad$ total number of suppliers

$N_{y} \quad$ total number of lines

$P_{\text {LOAD }} \quad$ initial active power of load demand [kW]

$P_{\text {MaxGen }}$ maximum generator' active power $[\mathrm{kW}]$

$P_{\text {MaxRed }}$ maximum consumer' reduction power $[\mathrm{kW}]$

$P_{\text {MaxSupplier }}$ maximum supplier' active power $[\mathrm{kW}]$

pr reserve use probability
$P_{\text {Required }}$ reserve product required power $[\mathrm{kW}]$

QMaxGen maximum generator reactive power [kVAr]

$Q_{\text {MaxSupplier maximum supplier reactive power }[\mathrm{kVAr}]}$

$Q_{\text {LOAD }} \quad$ initial reactive power of load demand $[k V A r\rfloor$

$T$ total number of hours in the time horizon [h]

$\bar{y} \quad$ series admittance of line that connect [S]

$\overline{y_{\mathrm{Sh}}} \quad$ shunt admittance of line connected in the bus [S]

Variables

$P \quad$ active power $[\mathrm{kW}]$

$Q \quad$ reactive power [kVAr]

$S \quad$ apparent power flow [kVA]

$\bar{U} \quad$ voltage in polar form [V]

$V \quad$ voltage magnitude [V]

X binary variable

\section{Subscript \\ Gen generator \\ max upper bound limit \\ $\min \quad$ lower bound limit \\ Supplier supplier

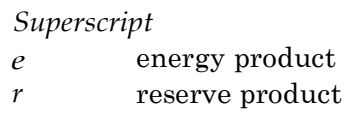

GCP generation curtailment power [kW]

NSD non-supplied demand [kW]

Red consumption reduction $[\mathrm{kW}]$ market strategy. Moreover, the participation in electricity markets requires annual fees creating barriers to the small resources. Due to the distributed characteristic of the referred resources, the aggregator should be able to aggregate resources from several geographic areas.

Taking into account these aspects, new entities have appeared in the power systems sector, as the case of VPPs, which enable smallsize wind farms to sell electricity in the market, while being aggregated with other wind farms, sharing the revenues obtained by the VPP for their market participation [10]. VPPs (acronym traditionally used to represent Virtual Power Plants and lately referring to Virtual Power Players [10]) are able to aggregate several types of distributed energy resources, such as DG, storage, DR and, most recently, electric vehicles. Otherwise, small players would not be able to participate actively in electricity markets due to their reduced power capacity and to their reduced technical means to implement an economic strategy.

From another point of view, and focusing on the work presented in this paper, the VPP can manage a specific distribution network area, managing the available resources connected to its network in order to achieve the energy and reserve needs. Eventually, the referred VPP can manage other energy resources in order to participate in electricity markets.

Another important issue, regarding DG and DR resources operation, is their availability intermittence and unpredictability. In this context, adequate concerns must be given to the specification of power reserve (ancillary services) at several levels of power systems operation, in order to maintain the expected increased levels of security in their operation. In this way, the provision of ancillary services is not only an additional necessity, but also an opportunity for DG, DR, and VPPs to participate in electricity markets [5,11e13] address the integration of DG, including the storage in electricity markets.
One of the late and most relevant works of the literature regarding dispatch of energy and reserve considering DR, presented in Ref. [14] makes use of DR for reserve provision and peak shaving, including the selling of the available capacity in their distinct DR utilization patterns. The optimization problem is a unit commitment which considers the demand and generators providing energy and reserve.

With regards to the system contingences, the authors of [15] considered the participation of demand in reserve provision by a voluntary reduction of demand, focusing on the recovery period.

In what concerns the incorporation of reliability standards in the economic dispatch of joint energy and ancillary services markets, Ref. [16] illustrates its application to the standards on DR.

The present paper presents an evolution of the work published in Ref. [17] by the same authors, and proposes a methodology in which a VPP aggregates several distributed energy resources, including DR and DG, and the energy acquired to electricity suppliers, in order to fulfill their electricity needs. The VPP operates a distribution network, performing the economic and technical management to minimize the operation costs. The VPP operation of the distribution network also considers the evaluation of network constraints in the scope of a determined resources' scheduling result, and the probability of the established amount of reserve being used in the system operation. These are the main contributions of the present paper. The results of the optimization model obtained in this paper for a case study of 218 consumers can include the energy that the VPP has contracted to deliver to the electricity market. This contracted energy regards the energy and reserve participation in the mentioned electricity market.

After this introduction section, Section 2 details the main contributions of the paper and explains the proposed methodology. Then, Section 3 presents the mathematical formulation of the optimization problem. Section 4 presents an illustrative case study 
which considers a 32-bus distribution network with 218 consumers and 66 DG units. The results obtained with the application of the proposed methodology to the selected case study are presented in Section 5. Finally, the most important conclusions of the work are presented in Section 6.

\section{Proposed energy and reserve dispatch methodology}

This section explains the proposed methodology and details the main contributions of the work in the field of joint energy and reserve dispatch.

The electrical energy negotiated in an electricity market can be divided into two distinct products e the energy and the reserve [11]. Generators traditionally ensured both products and recently DR programs have also been used to ensure them. The use of DR for reserve requirement fulfillment is very interesting due to the adequacy of the DR fast response [18]. Moreover, the reserve is mostly important in periods of peak power, when a contingency can cause increased impact.

A Virtual Power Player (VPP) can have several specific classifications concerning the core of its activities [10]. It is possible to find VPPs oriented for generation, demand response, electric vehicles, storage systems, or for a mix of resources management. However, VPPs have a common purpose which is to aggregate resources for the participation in competitive environments, namely in electricity markets. After defining bids' price and power resulting from the remuneration of the aggregated resources and by the VPP strategy in order to improve their profits, the VPP is able to submit these bids to several electricity markets' sessions. Market mechanisms and architecture are not addressed in the present paper, since the focus is given to the aggregated resources side, and, as referred, the defined bids can be flexibly submitted to market sessions or used in bilateral negotiations.

The VPP addressed in the present paper is also responsible for the technical operation of the distribution network. So, in this case, the bids that each single resource submits to the VPP are scheduled as defined in the optimization model. This means that each resource, once scheduled, will be remunerated at the submitted bid price. After that, the VPP manages the available resources in order to achieve the owned network requirements. These concern demand satisfaction and the reserves required to maintain adequate stability and reserve levels namely in order to address the variability of wind resources.

Currently, electricity markets consider energy and reserves as key products for their operation with appropriate levels of security and reliability [19]. In real markets, the procurement of these products is essentially based on two kinds of methodologies. For instance, MIBEL [20] operates with separate schedule of energy and reserves. In CAISO energy and reserves are jointly scheduled [21]. In the present paper, VPP addresses the energy and reserve needs in its area using a joint energy and reserve optimization schedule. Besides the energy and the reserve needs, the procurement can include additional quantities to be sold in the electricity market (see Fig. 1).

The proposed methodology considers that the VPP receives separated bids (remuneration prices and maximum power) for energy and for reserve fulfillment from each distinct generation and demand resource. This makes possible to address the concerns on the resources operation prices and also on the operation requirements that each resource can have (for example, wind generators operation requires adequate reserves due to its output power variability).

In this way, the resources schedule used in the mentioned products is performed by a VPP aiming to minimize the operation costs of supplying the demand and to ensure the adequate reserve.

The related literature works lack considering the DG in the joint scheduling of energy and reserve, as well as considering the VPPs,

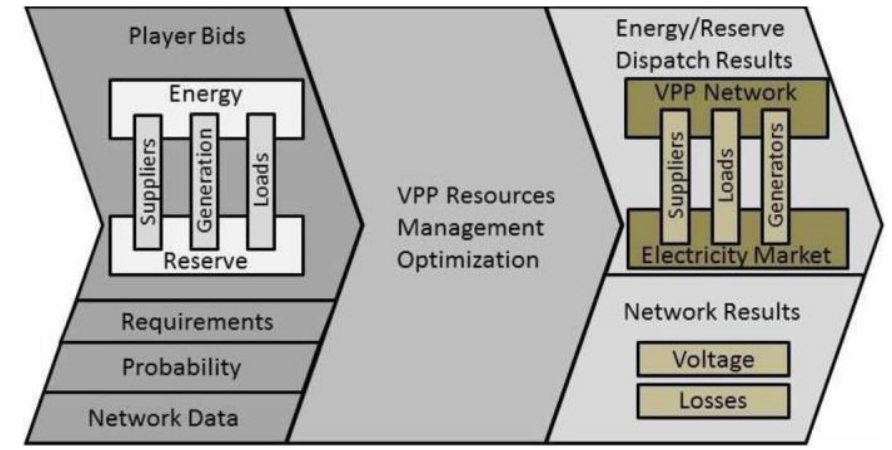

Fig. 1. Proposed methodology diagram.

External suppliers that can supply electricity through the connections between the VPP's owned network and the larger distribution network can also present bids for the participation in the energy resources schedule.

The proposed methodology considers the VPP operation and its inherent context characteristics [10]. In this way, the methodology considers a context of intensive penetration of DR and DG resources in distribution networks. Furthermore, in the considered future scenario of competitive electricity market environments, even at distribution network levels, in the scope of the operation of a VPP, DR participants and DG units should be able to compete in electricity markets (in an aggregated way). In this context, it is considered that incentives for DG are not available any longer; for example, the current use of feed-in-tariffs is not considered. However, it is considered that the DR resources should be able to technically provide the scheduled consumption reduction in the required time constraints. It is assumed that the VPP and each resource define the remuneration price that better fits the interest of both entities. In the proposed methodology, in the case of the reserve fulfillment, each resource once scheduled is remunerated by the fixed costs component; linear and quadratic cost components are applied if the resource is actually used.

From the suppliers side, the VPP operation activities consider that energy can be bought by the VPP to several suppliers at a fixed price for each period or for long periods as agreed, namely in the context of bilateral contracts. Using these contracts, the VPP operation proposed model is able to consider the use of the contracted power and price in order to integrate it in the optimal resources schedule in the owned distribution network. The proposed model also considers that the suppliers (which can be, for example, retailers) can supply energy at distinct prices for energy and for reserve. Using the proposed methodology, and having established energy acquisition contracts with suppliers, the VPP is able to perform the optimal resources schedule considering all the available resources and entities (DG, DR and suppliers).

The methodology proposed and developed by the authors is innovative and makes the following contributions on the joint energy and reserve dispatch including DR:

- It includes several types of DG as resources, competing with DR and electricity suppliers, for the energy and reserve provision;

- It focuses on a VPP that manages a distribution network to obtain the energy and reserve to fulfill its own needs and the contracted energy to be supplied to the electricity market;

- The formulation of the optimization problem hereby presented includes network constraints in order to technically validate the economic solution obtained by the model;

- It considers the probability of using the required reserve, as well as the required amount of reserve itself. operating energy and reserve capacities. The way the probability of using the reserve is considered is this paper is also innovative.

The interest of the proposed methodology relies on the need for a 
VPP to consider the provision of reserves, from an economic pointof view, in the two cases. Firstly, as the VPP operates the distribution network, and all the available DG resources, it must take into account the variability of the DG resources. This is the case of wind generators power, which unpredictability should be internally compensated, whereas sometimes the additional energy acquired from the upstream network (suppliers) has a high price. Secondly,

as the VPP should consider the participation in electricity markets, it needs to obtain reserve to participate in reserve-oriented markets, such as the case of ancillary services markets. From a technical point of view, the economic solution obtained for the resources' use needs to be validated in order to ensure the adequate power system stability.

Fig. 1 shows the schematic diagram of the proposed methodology resources managed by the VPP (generation and loads) and also the available suppliers and their current prices. The "VPP Resources

Management Optimization" module addresses the optimization problem considering the requirements for each product.

\section{Mathematical formulation}

The proposed problem aims to minimize the VPP costs and it can

be modeled as an optimization problem. The characteristics of the problem lead to a mixed-integer non-linear model and the problem consists on the minimization of a multimodal function with many local minima and a global optimum.

The objective function can be expressed as in Eq. (1). This objective function leads to the minimization of the costs considering the bids for energy and reserve products, made by suppliers, generators, and DR. All the bids are made with quadratic cost functions.

The consumers can be enrolled in the DR programs, in which each consumer has contracted with the VPP the consumption reduction amount and the respective price. In this way, the price paid for DR is agreed between VPP and the consumers. In another perspective, in the case that the VPP, due to any reason, is not able to supply all the actual demand, even using the scheduled reserves, the envisaged consumers will have Non-Supplied Demand (NSD) e cometimes referred as Energy Not Served (ENS) [19] e and must be

As previously referred, the Virtual Power Player is an aggrega-

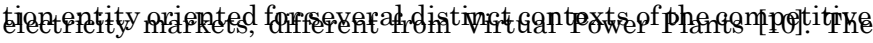

type of VPP considered in the proposed methodology owns a spe cific distribution network, being responsible for the economic and

technical operation of the network and the available and aggre-

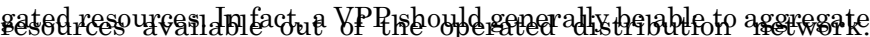

However, in the proposed methodology, since network constraints (related to load flow issues) are considered, all the aggregated re-

sources belong to that network.

The implemented resources optimization model considers that the reserves can be obtained in the aggregated resources, but also

from the upstream network, as it is economically more advantageous. In this way, the costs of the reserve, as well as the costs of energy, are evaluated in each specific period and the resources are scheduled accordingly so that VPP operation costs are minimized. All the resources (upstream network, DG and DR) are used in a flexible way, in order to address economic issues and technical issues like the accomplishment of the wind fluctuations. The model also considers the voltage and thermal limits in each bus and line, respectively.

When the resources schedule is performed, it is not possible to know about the effective use of the determined required power for reserve. Therefore, the probability of using the reserve is included in the model. The binary variables are due to the fact that the fixed costs only have to be considered when the resource is actually used. The linear costs related to the non-supplied demand and to the generation curtailment power are also included.

\section{7}

$$
\begin{aligned}
& \text { Minimize }
\end{aligned}
$$

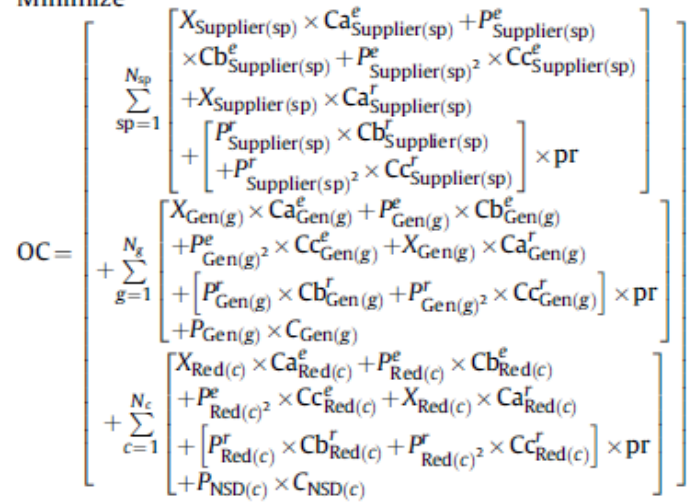

Regarding the constraints of the problem, one must guarantee the use of resources below their upper limits. The maximum curtailment of loads and the maximum production of distributed generation units and suppliers is modeled by Eqs. (2)e(16). Additionally, the power balance must be considered Eqs. (17)e(20). For each resource, there is a maximum quantity for participating in each product and a total capacity. These maxima are defined as a parameter of the bid submitted by the resource.

Equations (2) and (3) represent the maximum limit for the generation capacity of each supplier, for the energy and for the reserve. However, the sum of energy and reserve generation provided by the supplier should not exceed the upper limit of the production capacity, as shown in Eq. (4). The respective constraints regarding the reactive power are in Eqs. (5)e(7).

$$
\begin{aligned}
& P_{\text {Supplier(sp) }}^{e} \leq P_{\text {MaxSupplier(sp) }}^{e}, \quad \forall \mathrm{sp} \in\left\{1, \ldots, N_{\text {sp }}\right\} \\
& P_{\text {Supplier(sp) }}^{r} \leq P_{\text {MaxSupplier(sp) }}^{r}, \quad \forall \mathrm{sp} \in\left\{1, \ldots, N_{\text {sp }}\right\} \\
& P_{\text {Supplier(sp) }}^{r}+P_{\text {Supplier(sp) }}^{e} \leq P_{\text {MaxSupplier(sp) }}, \forall \mathrm{sp} \in\left\{1, \ldots, N_{\text {sp }}\right\} \\
& Q_{\text {Supplier(sp) }}^{e} \leq Q_{\text {MaxSupplier(sp) }}^{e}, \quad \forall \mathrm{sp} \in\left\{1, \ldots, N_{\text {sp }}\right\} \\
& Q_{\text {Supplier(sp) }}^{r} \leq Q_{\text {MaxSupplier(sp) }}^{r}, \quad \forall \mathrm{sp} \in\left\{1, \ldots, N_{\text {sp }}\right\} \\
& Q_{\text {Supplier(sp) }}^{r}+Q_{\text {Supplier(sp) }}^{e} \leq Q_{\text {MaxSupplier(sp) }}, \quad \forall \mathrm{sp} \in\left\{1, \ldots, N_{\text {sp }}\right\}
\end{aligned}
$$

Similarly to the previous constraints, Eqs. (8)e(10) refer to the upper limits of active power generation applied to DG generators, while Eqs. (11)e(13) regard the upper limits of DG generators' 
reactive power generation. Equations (14)e(16) concern the active power demand reduction upper limits of DR resources.

$$
\begin{aligned}
& P^{e} \quad P_{\text {Gen }(g)}^{e} \leq P_{\text {MaxGen }(g)}^{e}, \quad \forall g \in\left\{1, \ldots, N_{g}\right\} \\
& P_{\text {Gen }(g)}^{r} \leq P_{\text {MaxGen }(g)}^{r}, \quad \forall g \in\left\{1, \ldots, N_{g}\right\} \\
& \text { ) } \\
& P_{\mathrm{Gen}(g)}^{r}+P_{\mathrm{Gen}(g)}^{e} \leq P_{\operatorname{MaxGen}(g)}, \quad \forall g \in\left\{1, \ldots, N_{g}\right\} \\
& Q^{e} \\
& Q_{G e n(g)}^{e} \leq Q_{M a x G e n(g)}^{e}, \quad \forall g \in\left\{1, \ldots, N_{g}\right\} \\
& Q_{G \operatorname{Gen}(g)}^{r} \leq Q_{\operatorname{MaxGen}(g)}^{r}, \quad \forall g \in\left\{1, \ldots, N_{g}\right\} \\
& Q^{r} \\
& Q_{G \operatorname{Gen}(g)}^{r}+Q_{G e n(g)}^{e} \leq Q_{\operatorname{MaxGen}(g)}, \quad \forall g \in\left\{1, \ldots, N_{g}\right\} \\
& P_{\operatorname{Red}(c)}^{e} \leq P_{\operatorname{MaxRed}(c)}^{e}, \quad \forall c \in\left\{1, \ldots, N_{c}\right\} \\
& P_{\operatorname{Red}(c)}^{r} \leq P_{\operatorname{MaxRed}(c)}^{r}, \quad \forall c \in\left\{1, \ldots, N_{c}\right\} \\
& P_{\operatorname{Red}(c)}^{r}+P_{\operatorname{Red}(c)}^{e} \leq P_{\operatorname{MaxRed}(c)}^{e}, \quad \forall c \in\left\{1, \ldots, N_{c}\right\}
\end{aligned}
$$

There are four power balance equations. The first one Eq. (17) is the balance of each consumer's power, with the non-supplied power in the consumer. The second one Eq. (18) is the balance of all the resources participating in the reserve product, which need to guarantee the required power for this product. The third one Eq. (19) is the load-generation balance in the system, which considers non-supplied power in each consumer, as well as the generation curtailment power by each DG generator. The last one Eq. (20) is the network reactive power balance in each period $t$ and in each bus $b$.

$$
\begin{aligned}
& P_{\operatorname{Red}(c)}^{\mathrm{r}}+P_{\operatorname{Red}(c)}^{e}+P_{\mathrm{NSD}(c)} \leq P_{\mathrm{Load}(c)}, \forall c \in\left\{1, \ldots, N_{c}\right\} \\
& \sum_{s p=1}^{N_{\text {sp }}} P_{\text {Supplier(sp) }}^{r}+\sum_{g=1}^{N_{g}} P_{G e n}^{r}(g)+\sum_{c=1}^{N_{\varepsilon}} P_{\text {Red }(c)}^{r}=P_{\text {Required }}^{r} \\
& \sum_{s p=1}^{N_{s p}^{i}}\left[P_{\text {Supplier(sp) }}^{e, i}\right]+\sum_{g=1}^{N_{g}^{e}}\left[P_{G \text { Gen }(g)}^{e, i}+P_{\mathrm{EGP}(g)}^{i}\right]+\sum_{c=1}^{N_{c}^{i}}\left[P_{\operatorname{Red}(c)}^{e, i}\right. \\
& \left.+P_{\mathrm{NSD}(c)}^{i}-P_{\mathrm{Laad}(c)}^{i}\right]=\sum_{j=1}^{N_{b}}\left[V_{i(t)} \times V_{j(t)}\left(G_{i j} \cos \left(\theta_{i(t)}-\theta_{j(t)}\right)\right.\right. \\
& \left.\left.+B_{i j} \sin \left(\theta_{i(t)}-\theta_{j(t)}\right)\right)\right] \quad \forall t \in\{1, \ldots, T\} ; \forall i \in\left\{1, \ldots, N_{B}\right\}
\end{aligned}
$$

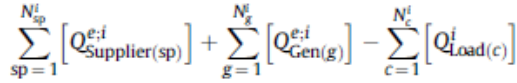

$$
\begin{aligned}
& =\sum_{j=1}^{N_{b}}\left[V_{i(t)} \times V_{j(t)}\left(G_{i j} \sin \left(\theta_{i(t)}-\theta_{j(t)}\right)-B_{i j} \cos \right.\right. \\
& \left.\left.\left(\theta_{i(t)}-\theta_{j(t)}\right)\right)\right] \quad \forall t \in\{1, . ., T\} ; \quad \forall i \in\left\{1, \ldots, N_{\mathrm{B}}\right\}
\end{aligned}
$$

\section{$\backslash \mathbf{i}$}

The network constraints are modeled by Eqs. (21)e(23). The bus voltage magnitude and angle limits are represented by Eqs. (21) and (22) respectively. For the slack bus, the voltage angle and magnitude are fixed and defined by the user. The line thermal limits constraint Eq. (23) is essential to guarantee the maximum power that can flow in a line.

$$
\begin{aligned}
& V_{i}^{\min } \leq V_{i(t)} \leq V_{i}^{\max } \quad \forall t \in\{1, \ldots, T\} ; \quad \forall i \in\left\{1, \ldots, N_{B}\right\} \\
& \theta_{i}^{\min } \leq \theta_{i(t)} \leq \theta_{i}^{\max } \quad \forall t \in\{1, \ldots, T\} ; \forall i \in\left\{1, \ldots, N_{B}\right\} \\
& \left|\overline{U_{i(t)}} \times\left[\overline{y_{i j}} \times\left(\overline{U_{i(t)}}-\overline{U_{j(t)}}\right)+\overline{y_{\text {sh_i }}} \times \overline{U_{j(t)}}\right]^{*}\right| \\
& \leq S_{\text {Ly }}^{\max } \quad \forall t \in\{1, \ldots, T\} ; \quad \forall i, j \in\left\{1, \ldots, N_{B}\right\} ; \quad i \neq j ; \quad \forall k \in\left\{1, \ldots, N_{y}\right\}
\end{aligned}
$$

The optimization problem presented in this section has been solved in GAMS [22].

\section{Case study}

This section presents a case study, which uses data of the network scenario initially presented in Ref. [23], which has been updated regarding the number of consumers in the network and their characterization. In the present case study, the 32-bus distribution network depicted in Fig. 2 accommodates 66 DG units, 218 consumers and 5 suppliers connected to bus 0 , which is the connection point between this network and the upstream network.

Fig. 2 also includes data concerning the amount and type of DG in each bus. The total consumption in each bus, as well as the consumption regarding each one of the six defined consumer types, is presented in Table 1. In this way, each one of the 218 consumers is connected to one of the 32 buses of the network and each type of consumer is characterized. The considered types of consumers are: DomesticeDM; Small CommerceeSC; Medium CommerceeMC; Large CommerceeLC; Medium IndustryeMI; and Large IndustryeLI.

In this case study, all the generators are offering the total available or installed capacity (a total of $2663 \mathrm{~kW}$ by the DG and $5500 \mathrm{~kW}$ from the suppliers), as shown in Table 2. For the sake of simplicity, it is assumed that each DG and each supplier offers $70 \%$ of its capacity to the energy prodjct; the remaining $30 \%$ regards the
participation in the reserve product.

The bid price of the generators is considered equal for all the generators of the same type. The proposed methodology considers that each consumer presents an initial expected consumption. Taking into account the referred value, each consumer is able to provide an amount of demand consumption reduction in order to participate in DR programs. In the present case study, it was assumed that each consumer is able to reduce the consumption by $40 \%$ of the initial expected consumption, for participation in DR programs.

The participation in DR programs considers, in the proposed methodology, two specific situations according to the context of VPPs operation: participating in the provision of energy capacity (competing side by side with generators); and providing reserve capacity to be used as needed by the VPP. This assumption is based on the FERC order 719, which requires that bids from demand response resources should be accepted on a basis comparable to any other resources [24]. Equations (19) and (20) include the energy balance between the initial expected consumption and the two DR prodycts (energy and reserve ipeach consumer. In the nsumer is $60 \%$ and $40 \%$ of the consumption reduction capacity

( $24 \%$ and $16 \%$ of the initial expected consumption), respectively for energy and reserve products.

Table 3 presents the values of consumer bid prices. These values are considered equal for the consumers of the same type for the 


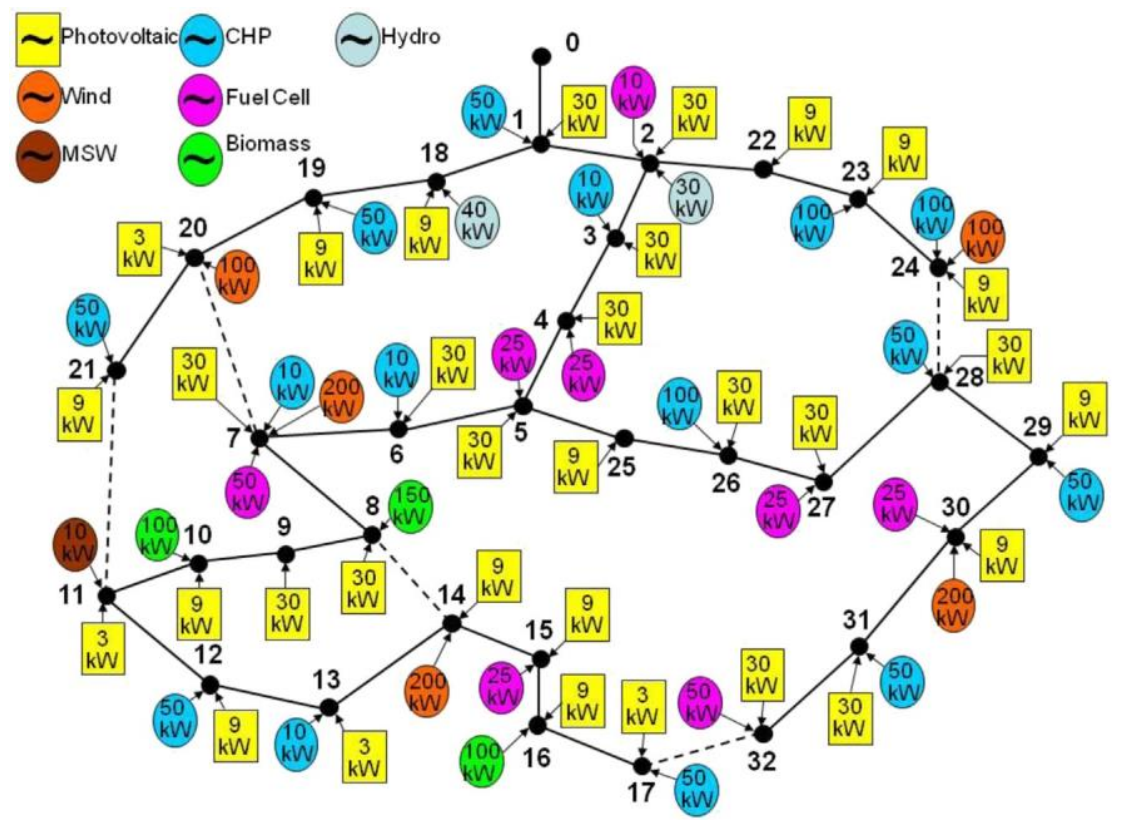

Fig. 2. Distribution network.

energy and reserve products. For both products, consumer bids consider quadratic energy cost functions. The consumers scheduled for the participation in one or both products are remunerated at the price they bid. The characterization data of the network elements can be found in Ref. [25].

\section{Results}

The present section shows the results obtained by the application of the proposed methodology to the case study presented in Section 4 . The present section is divided into three subsections:

Table 1

Active power demand in each bus.

\begin{tabular}{|c|c|c|c|c|c|c|c|c|c|c|c|c|c|}
\hline \multirow[t]{2}{*}{ Bus } & \multirow[t]{2}{*}{ Demand (kW) } & \multicolumn{6}{|c|}{ Power consumption (\%) } & \multicolumn{6}{|c|}{ Number of consumers } \\
\hline & & $\mathrm{DM}$ & $\mathrm{SC}$ & $\mathrm{MC}$ & $\mathrm{LC}$ & MI & LI & $\mathrm{DM}$ & $\mathrm{SC}$ & $\mathrm{MC}$ & $\mathrm{LC}$ & MI & LI \\
\hline 1 & 169.0 & e & 20 & 40 & 40 & e & e & e & 2 & 2 & 1 & e & e \\
\hline 2 & 148.0 & 25 & 75 & e & e & e & e & 2 & 5 & e & e & e & e \\
\hline 3 & 147.0 & 40 & 60 & e & e & e & e & 4 & 4 & e & e & e & e \\
\hline 4 & 145.0 & 70 & 30 & e & e & e & e & 7 & 2 & e & e & e & e \\
\hline 5 & 94.0 & 100 & e & e & e & e & e & 8 & e & e & e & e & e \\
\hline 6 & 311.0 & 20 & 10 & e & 70 & e & e & 4 & 1 & e & 2 & e & e \\
\hline 7 & 309.0 & e & 10 & 20 & 70 & e & e & e & 1 & 1 & 2 & e & e \\
\hline 8 & 89.0 & 85 & 15 & e & e & e & e & 9 & 1 & e & e & e & e \\
\hline 9 & 91.0 & 100 & e & e & $e$ & e & e & 10 & e & e & e & e & e \\
\hline 10 & 67.0 & 60 & 40 & e & e & e & e & 4 & 2 & e & e & e & e \\
\hline 11 & 91.0 & 80 & 20 & e & e & e & e & 6 & 1 & e & e & e & e \\
\hline 12 & 91.0 & 100 & e & e & e & e & e & 7 & e & $e$ & e & e & e \\
\hline 13 & 181.0 & 30 & 20 & 50 & $e$ & e & e & 5 & 2 & 2 & e & $e$ & e \\
\hline 14 & 91.0 & 100 & e & e & e & e & e & 6 & e & e & e & e & e \\
\hline 15 & 91.0 & 80 & 20 & e & e & e & e & 7 & 1 & e & e & e & e \\
\hline 16 & 92.0 & 65 & 35 & e & e & e & e & 5 & 2 & e & e & e & e \\
\hline 17 & 135.0 & 15 & 60 & 25 & $e$ & e & $e$ & 2 & 4 & 1 & $e$ & $e$ & e \\
\hline 18 & 152.0 & e & e & 30 & 70 & e & e & e & e & 2 & 2 & e & e \\
\hline 19 & 152.0 & 15 & e & 50 & 35 & e & e & 3 & e & 3 & 1 & e & e \\
\hline 20 & 152.0 & e & 40 & 60 & e & e & e & e & 4 & 4 & e & e & e \\
\hline 21 & 151.0 & e & 20 & 40 & 40 & e & e & e & 2 & 2 & 1 & e & e \\
\hline 22 & 147.0 & 20 & 80 & e & e & e & e & 2 & 5 & e & e & e & e \\
\hline 23 & 675.0 & 5 & 5 & e & e & e & 90 & 2 & 1 & e & e & e & 4 \\
\hline 24 & 669.0 & e & 5 & e & e & 10 & 85 & e & 1 & e & e & 1 & 4 \\
\hline 25 & 94.0 & 100 & e & e & e & e & e & 7 & e & e & e & e & e \\
\hline 26 & 93.0 & 75 & 25 & e & e & e & e & 5 & 1 & e & e & e & e \\
\hline 27 & 92.0 & 100 & e & e & e & e & e & 8 & e & e & e & e & e \\
\hline 28 & 183.0 & 15 & 25 & 60 & e & e & e & 2 & 2 & 3 & e & e & e \\
\hline 29 & 295.0 & e & 10 & 15 & e & 75 & e & e & 1 & 1 & e & 3 & e \\
\hline 30 & 225.0 & e & 10 & e & e & 60 & 30 & e & 1 & e & e & 3 & 1 \\
\hline 31 & 315.0 & e & e & 20 & 80 & e & e & e & e & 2 & 4 & e & e \\
\hline 32 & 90.0 & 100 & e & e & e & e & e & 5 & e & e & e & e & e \\
\hline Total & 5827.0 & e & e & e & e & e & e & 120 & 46 & 23 & 13 & 7 & 9 \\
\hline
\end{tabular}


Table 2

Generators' characteristics and bid prices.

\begin{tabular}{|c|c|c|c|c|c|c|c|c|c|c|}
\hline \multirow[t]{2}{*}{ Type of generator } & \multirow{2}{*}{$\begin{array}{l}\text { Number of } \\
\text { units }\end{array}$} & \multirow{2}{*}{$\begin{array}{l}\text { Minimum } \\
\text { capacity } \\
(\mathrm{kW})\end{array}$} & \multirow{2}{*}{$\begin{array}{l}\text { Maximum } \\
\text { capacity } \\
(\mathrm{kW})\end{array}$} & \multirow{2}{*}{$\begin{array}{l}\text { Total } \\
\text { capacity } \\
(\mathrm{kW})\end{array}$} & \multicolumn{3}{|l|}{ Energy } & \multicolumn{3}{|l|}{ Reserve } \\
\hline & & & & & $a(\mathrm{~m} . \mathrm{u} . / \mathrm{h})$ & $b(\mathrm{~m} . \mathrm{u} . / \mathrm{kWh})$ & $c\left(\mathrm{~m} . \mathrm{u} . / \mathrm{kWh}^{2}\right)$ & $a(\mathrm{~m} . \mathrm{u} . / \mathrm{h})$ & $b(\mathrm{~m} . \mathrm{u} . / \mathrm{kWh})$ & $c\left(\mathrm{~m} \cdot \mathrm{u} . / \mathrm{kWh}^{2}\right)$ \\
\hline Photovoltaic & 32 & 3 & 30 & 558 & 0 & 0.15 & 0 & 0 & 0.165 & 0 \\
\hline Cogeneration (CHP) & 15 & 10 & 100 & 740 & 0.000151 & 0.001062 & 0.001006 & 0.000166 & 0.001168 & 0.001106 \\
\hline Fuel cell & 8 & 10 & 50 & 235 & 0 & 0.098 & 0 & 0 & 0.1078 & 0 \\
\hline Hydro & 2 & 30 & 40 & 70 & 0 & 0.042 & 0 & 0 & 0.0462 & 0 \\
\hline Wind & 5 & 100 & 200 & 700 & 0 & 0.071 & 0 & 0 & 0.0781 & 0 \\
\hline Biomass & 3 & 100 & 150 & 350 & 0 & 0.086 & 0 & 0 & 0.0946 & 0 \\
\hline $\begin{array}{l}\text { Waste to energy } \\
\text { (MSW) }\end{array}$ & 1 & 10 & 10 & 10 & 0 & 0.056 & 0 & 0 & 0.0616 & 0 \\
\hline Supplier1 & 1 & e & 1200 & 1200 & 0 & 0.23 & 0 & 0 & 0.286 & 0 \\
\hline Supplier2 & 1 & e & 800 & 800 & 0 & 0.24 & 0 & 0 & 0.264 & 0 \\
\hline Supplier3 & 1 & e & 900 & 900 & 0 & 0.25 & 0 & 0 & 0.275 & 0 \\
\hline Supplier4 & 1 & e & 1800 & 1800 & 0 & 0.26 & 0 & 0 & 0.253 & 0 \\
\hline Supplier5 & 1 & e & 800 & 800 & 0 & 0.27 & 0 & 0 & 0.297 & 0 \\
\hline
\end{tabular}

Table 3

Consumers' characteristics and bid prices.

\begin{tabular}{|c|c|c|c|c|c|c|c|}
\hline \multirow[t]{2}{*}{ Type of consumer } & & \multicolumn{3}{|l|}{ Energy } & \multicolumn{3}{|l|}{ Reserve } \\
\hline & & $a(\mathrm{~m} . \mathrm{u} . / \mathrm{h})$ & $b(\mathrm{~m} . \mathrm{u} . / \mathrm{kWh})$ & $c\left(\mathrm{~m} . \mathrm{u} . / \mathrm{kWh}^{2}\right)$ & $a(\mathrm{~m} . \mathrm{u} . / \mathrm{h})$ & $b(\mathrm{~m} . \mathrm{u} . / \mathrm{kWh})$ & $c\left(\mathrm{~m} . \mathrm{u} . / \mathrm{kWh}^{2}\right)$ \\
\hline Domestic & $\mathrm{DM}$ & 0.0020 & 0.20 & 0.000020 & 0.0021 & 0.21 & 0.000021 \\
\hline Small Commerce & $\mathrm{SC}$ & 0.0016 & 0.16 & 0.000016 & 0.0018 & 0.18 & 0.000018 \\
\hline Medium Commerce & $\mathrm{MC}$ & 0.0019 & 0.19 & 0.000019 & 0.0020 & 0.20 & 0.000020 \\
\hline Large Commerce & $\mathrm{LC}$ & 0.0018 & 0.18 & 0.000018 & 0.0019 & 0.19 & 0.000019 \\
\hline Medium Industrial & MI & 0.0012 & 0.12 & 0.000012 & 0.0012 & 0.12 & 0.000012 \\
\hline Large Industrial & LI & 0.0014 & 0.14 & 0.000014 & 0.0007 & 0.07 & 0.000007 \\
\hline
\end{tabular}

Subsection 5.1 presents the results obtained when the probability of using the reserve is equal to 1 ; in Subsection 5.2, the influence of distinct probabilities of using the reserve is analyzed; finally, in Subsection 5.3, the results obtained in the network constraints validation are addressed.

\subsection{Probability of reserve use equal to 1}

The VPP operation costs in a specific situation depend on the required reserve amount, on the actually used reserve energy, and on the price of the resources.

Let us assume that the probability of using the reserve is equal to 1. The bidding resource use and the VPP operation costs are analyzed regarding the variations in the amount of power required for the reserve product and the variations in the price of electricity provided by the suppliers.

Fig. 3 presents the values of the objective function (operation costs) regarding the required reserve amount and the supplier price.

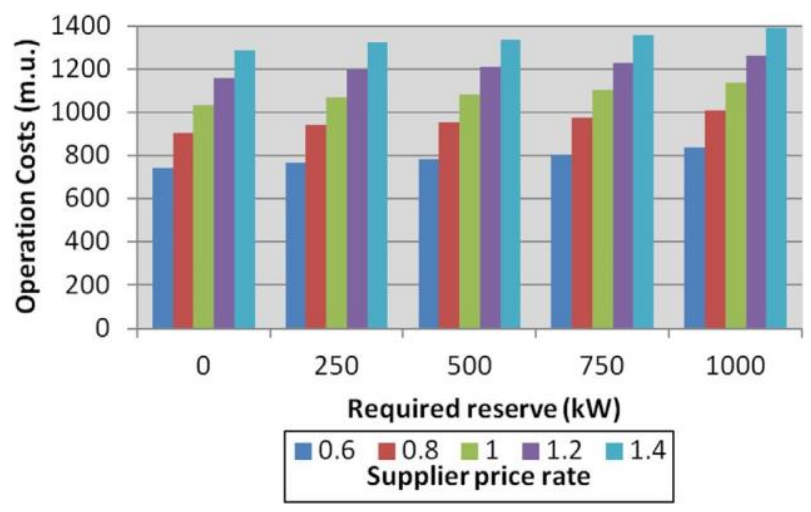

Fig. 3. Values of the objective function regarding the required reserve and the supplier price rate.
Taking into account the reference values (labeled as " 1 " in Fig. 3), several steps of supplier prices were defined (for example, step " 0.6 " corresponds to a reduction of $40 \%$ in the basis price; step " 1 " is the basis price; and step "1.4" corresponds to an increase of $40 \%$ in the suppliers price). One can see that the increase in the supplier price rate and in the required reserve cause an increase in the operation costs. Therefore, the supplier price rate significantly influences the operation costs.

Figs. 4 and 5 present the values of the DR use regarding the variations in the supplier price, respectively for the energy and reserve products. The results are organized by type of consumer and they consider a value of $750 \mathrm{~kW}$ for the amount of required reserve.

Regarding the participation of DR in the energy product, one can say that MI (medium industry) and LI (large industry) consumers always participate, regardless the supplier price rate. The consumers of other types do not participate when the supplier prices are reduced in $40 \%$.

In the results presented in Fig. 4, the total amount of DR participation is constant because the required reserve power is constant and equal to $750 \mathrm{~kW}$. In the case of the reserve product, only MI and LI consumers participate. This participation is independent of the supplier price rate.

Figs. 6 and 7 present the values of generation use regarding the variations in the supplier price, respectively for the energy and reserve products.

The results are organized by type of generation. Regarding the energy product, only the municipal solid waste (MSW) and supplier 5 do not participate. Depending on the supplier price rate, the amount of power used in each resource can vary. The special case of supplier 4 should be noted: its participation significantly increases when the supplier price is reduced in $40 \%$.

In the case of the reserve product, as the required amount is always the same, regardless the supplier price rate, the total amount of use is always the same. In this product, for the specified conditions, only the suppliers are scheduled. 


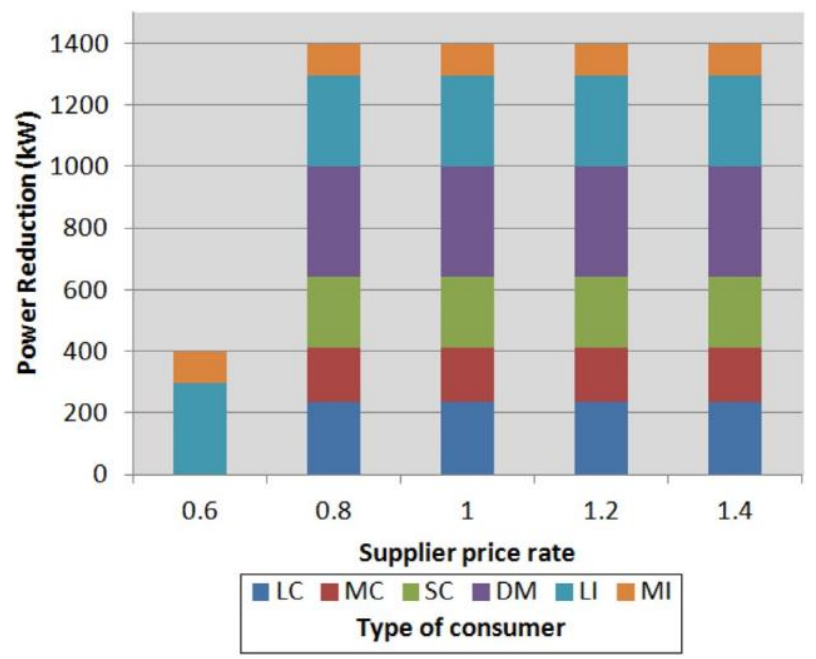

Fig. 4. DR use regarding the supplier price rate and the type of consumer, for the energy product.

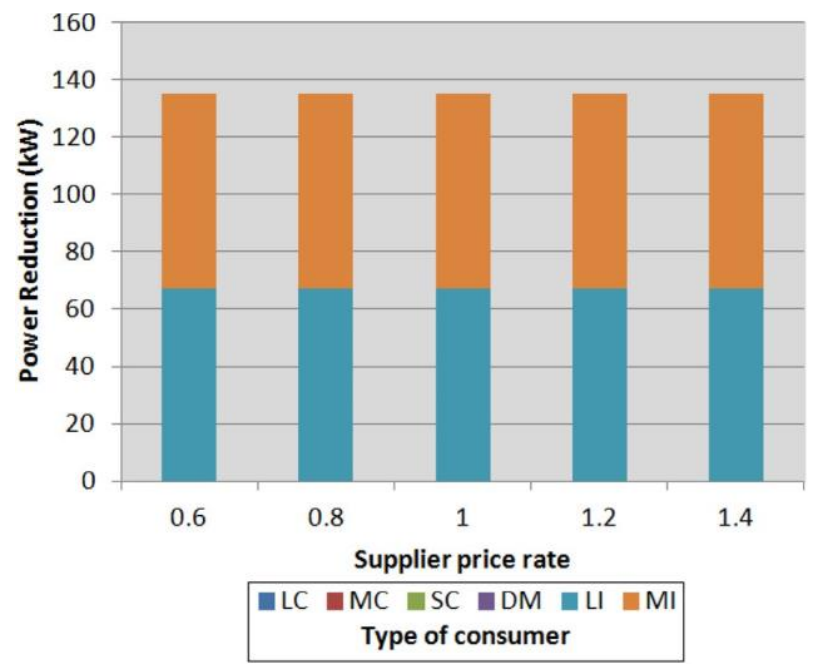

Fig. 5. DR use regarding the supplier price rate and the type of consumer, for the reserve product.

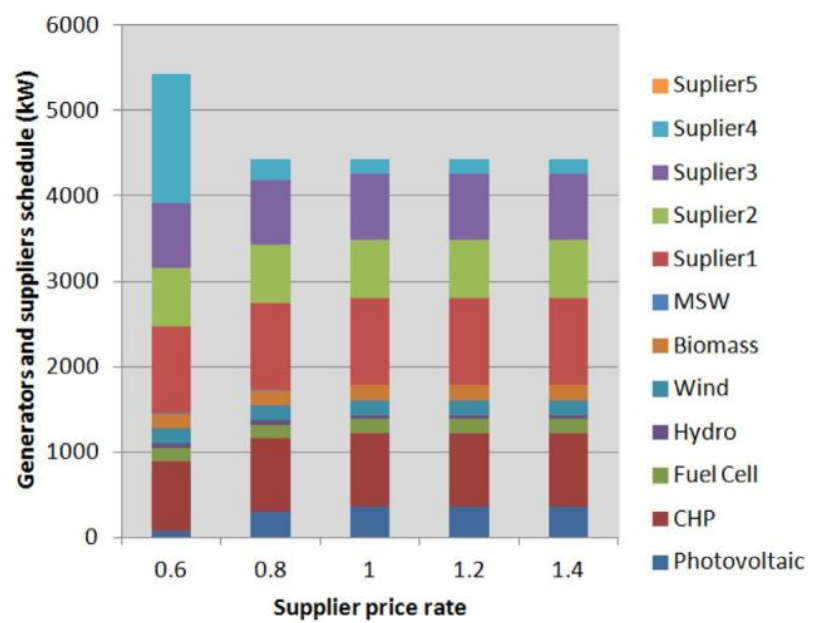

Fig. 6. Generation use regarding the supplier price rate for energy.

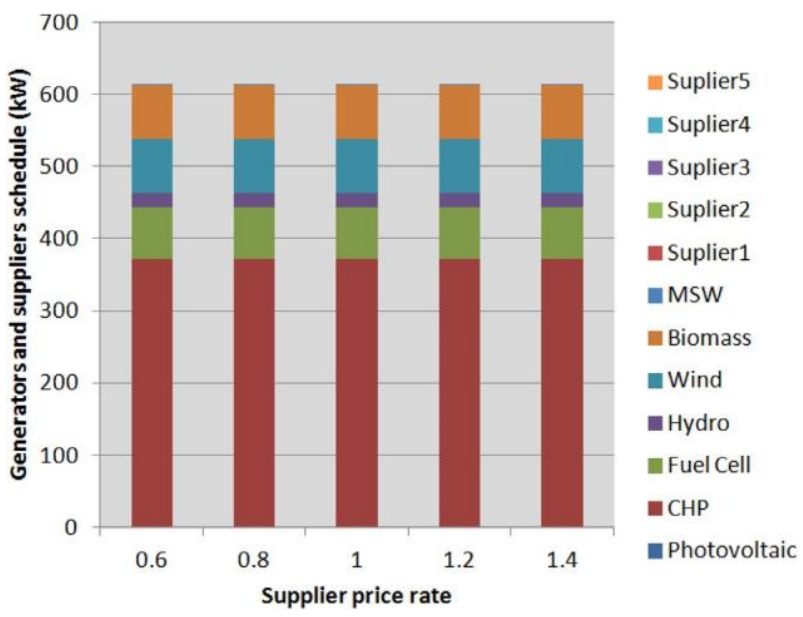

Fig. 7. Generation use regarding the supplier price rate for reserve.

\subsection{Variable probability of reserve use}

This subsection presents results in the perspective of analyzing the influence of the reserve use probability on the obtained costs and scheduling.

In order to evaluate the influence of the reserve use probability, some results are analyzed in the present subsection. The required reserve power is assumed to be equal to $750 \mathrm{~kW}$. Fig. 8 presents the values of the objective function regarding the variations in the reserve probability of use and the variations in the supplier price rate.

One can see that both increases in the supplier price rate and in the reserve probability of use cause an increase in the operation costs. In this situation, the supplier price rate significantly also influences the operation costs.

Figs. 9 and 10 show the use of the power generation and reduction, respectively for generation resources and for the consumers' response, regarding the variations in the supplier pricerate and in the reserve probability of use.

Fig. 9 shows the results of the generation and supplier power use in the reserve product. The value of the supplier price rate does not influence the total amount of generator and supplier resources usage. The value of probability of using the reserve only affects the amount of resources usage when that probability is zero.

Fig. 10 shows the results of DR usage in the same conditions used in Fig. 9. As concluded for the use of generator and supplier resources, the value of the supplier price rate does not influence the

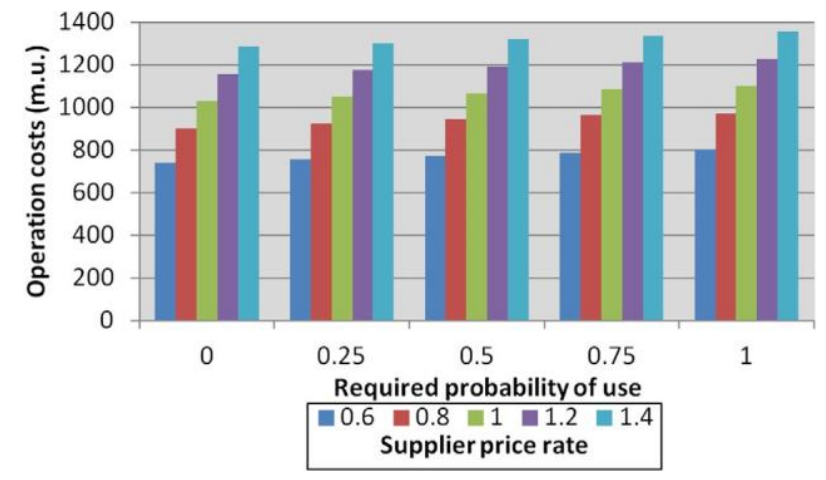

Fig. 8. Values of the objective function regarding reserve probability of use and sup plier price rate. 


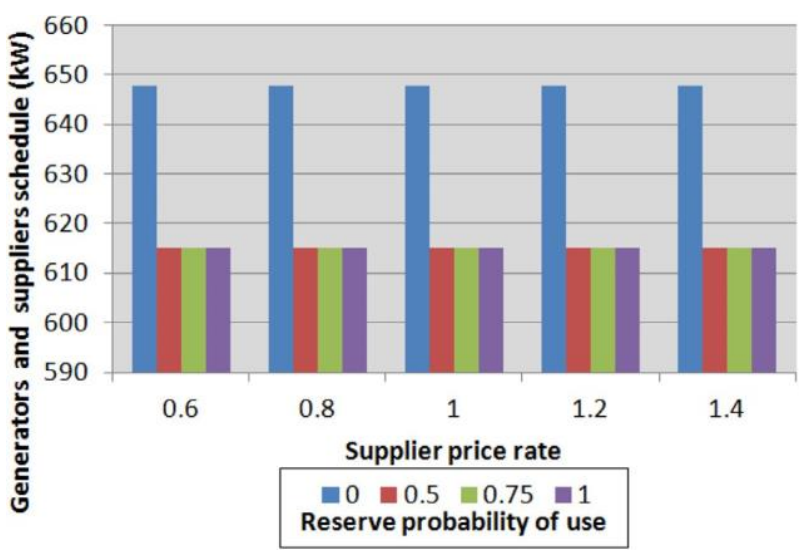

Fig. 9. Generation use regarding the supplier price rate and the reserve probability of use, in the reserve product.

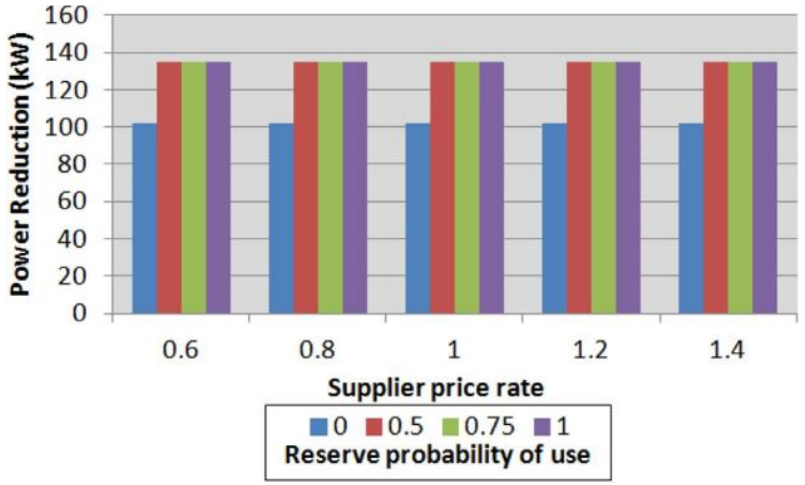

Fig. 10. DR use regarding the supplier price rate and reserve probability of use, for the reserve product.

amount of DR usage. Moreover, for the value of probability of using the reserve, it only affects the amount of resources usage when that probability is zero.

\subsection{Optimization problem}

The present subsection presents the technical results obtained with the proposed methodology, regarding the network constraints validation. Several voltage and losses values were selected to illustrate the results obtained.

Fig. 11 shows the bus voltage magnitude values, regarding several values of supplier price and using a need of reserve of $750 \mathrm{~kW}$, with a probability of using the reserve equal to 1 . It can be seen that the voltage values are within acceptable limits in all buses. However, when the supplier electricity price is very low, the voltages in the buses electrically distant from the upstream network connection are very low too. This is due to the fact that the DG is not dispatched and those units do not contribute to the increase of voltages values leveling among the buses of the network. As expected, the voltage in bus 18 is close to the voltage in bus 1 , due to the network topology.

When we analyze the effect that the amount of required reserve can have in the voltage profile, as shown in Fig. 12, it is possible to find that the voltage values are acceptable and they are not significantly influenced by the value of reserve required.

In what concerns the total power losses in the studied distribution network, by analyzing the influence of the supplier electricity price and of the required reserve, the results are presented in

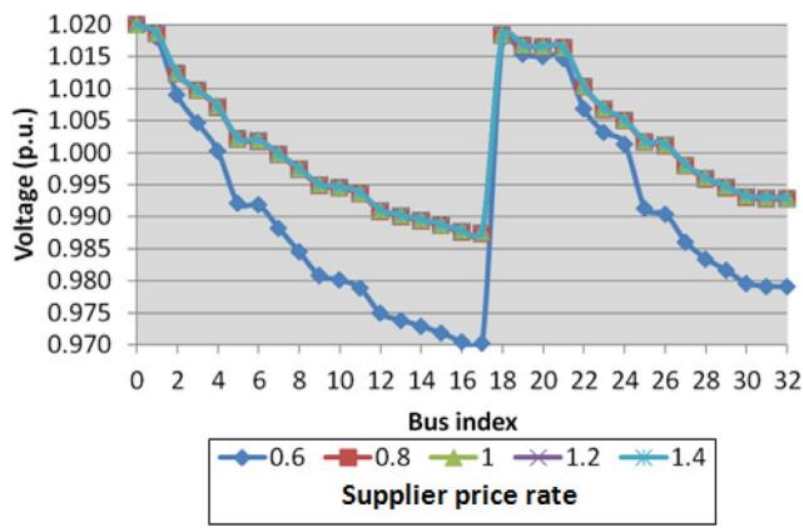

Fig. 11. Bus voltage magnitude regarding the supplier price rate.

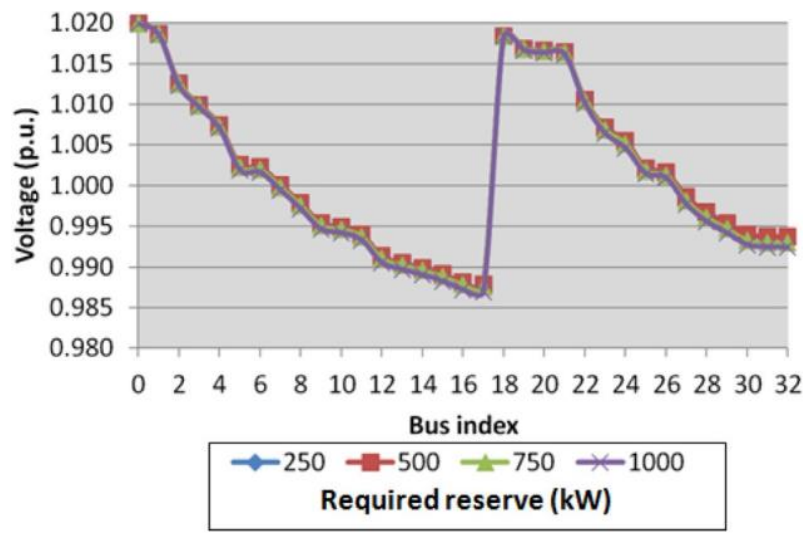

Fig. 12. Bus voltage magnitude regarding the amount of required reserve.

Fig. 13. It can be seen that an excessively low supplier electricity price cause an increase in the total power losses, due to the reduced use of distributed resources (DG and DR) in this situation. When the required energy is obtained locally, the network losses are reduced. This shows the importance of embedding the treatment of the network constraints in the proposed methodology. In this way, the interaction between technical and economic perspectives is addressed and lower operation costs can be obtained.

From Fig. 13, it can be concluded that the amount of required reserve, in the present scenario, does not have relevant impact in the value of power losses.

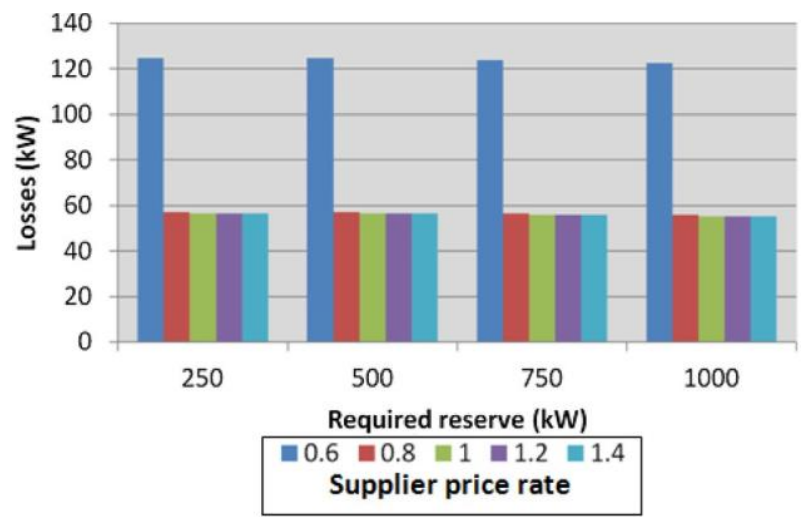

Fig. 13. Total network losses regarding the supplier price rate and the required amount of reserve. 


\section{Conclusions}

The implementation of electricity markets, together with the dissemination of new distributed small-size resources, as the case of demand response and distributed generation, has led to new challenges in the way that power systems are operated and planned. New players, such as the Virtual Power Players that aggregate generation and consumption resources, as well as the consumers themselves, are involved in this competitive environment. Moreover, increasing relevance should be given to the definition of reserve power in order to accommodate the variability and unpredictability of the availability of these new resources.

The methodology proposed in this paper considers the bids of demand response resources competing with the bids of generators and suppliers for both energy and reserve markets. The proposed method aims at minimizing VPP costs in the operation of a distribution network, considering the participation in the electricity market. In this way, the VPP is able to acquire energy to supply demand and to assure the required reserve as well as to participate in ancillary services markets. The probability of the reserve power being used is also addressed by the proposed methodology. Furthermore, the network constraints are included in the optimization model by power flow equations, in order to address the technical validation of the solution obtained for the optimal resources use.

A case study of a 32-bus distribution network with 66 distributed generation units, 218 consumers of 6 types, and 5 suppliers are included in the paper. The results considered several values for the probability of reserve use $(0 ; 0.25 ; 0.50 ; 0.75$ and 1$)$. It was possible to conclude that the VPP operation costs in a specific situation depend on the amount required for the reserve product and whether the reserve power is actually used or not.

It was also possible to conclude that the increase of the three varying parameters (the probability of using the reserve, the supplier price, and the required reserve amount) have a direct impact on the VPP operation costs. The highest relevance has been found for the impact of the supplier energy price.

In what concerns the network operation conditions, it was verified the negative technical impact of situations in which the supplier electricity price is rather low, where the losses in the network are increased and the voltage profile in the buses is worsened.

\section{Acknowledgments}

This work is supported by FEDER Funds through COMPETE program and by National Funds through FCT under the projects FCOMP-01-0124-FEDER: PEst-OE/EEI/UI0760/2011, PTDC/EEA-EEL/ 099832/2008, PTDC/SEN-ENR/099844/2008, and PTDC/SEN-ENR/ 122174/2010, and SFRH/BD/80183/2011 (P. Faria PhD).

\section{References}

[1] Kirschen D. Demand-side view of electricity markets. IEEE Trans Power Syst 2003;18:520e7.
[2] Charles River Associates. Primer on demand-side management with an emphasis on price-responsive programs, Washington D. C; 2005.

[3] Cappers P, Goldman C, Kathan D. Demand response in U.S. electricity markets: empirical evidence. Energy 2010;35:1526e35.

[4] Aalami H, Moghaddam M, Yousefi G. Demand response modeling considering interruptible/curtailable loads and capacity market programs. Appl Energy 2010;87:243e50

[5] Faria P, Vale Z. Demand response in electrical energy supply: an optimal real time pricing approach. Energy 2011;36:5374e84.

[6] Faria P, Vale Z, Soares J, Ferreira J. Particle swarm optimization applied to integrated demand response resources scheduling. In: IEEE symposium on computational intelligence applications in smart grid (CIASG); 11e15, 2011. pp. 1 e8.

[7] Woo C, Greening L. Special issue demand response resources: the US and international experience introduction. Energy 2010;35:1515e7.

[8] Conselho de Reguladores do MIBEL. Description of the operation of the MIBEL. visited on December 2013, available online: http://www.erse.pt/eng/ electricity/MIBEL/Documents/Description_Operation_MIBEL.pdf; November 2009.

[9] NordPool Spot. Elbas user guide. visited on December 2013, available online: http://www.nordpoolspot.com/Global/Download\%20Center/Elbas/Elbas-3.1 user-manual.pdf; October 2012.

[10] Morais H, Pinto T, Vale Z, Praça I. Multilevel negotiation in Smart grids for VPP management of distributed resources. IEEE Intell Syst 2012;27(6):8e16.

[11] Angelino R, Bracale A, Carpinelli G, Mangoni M, Proto D. A fuel cell-based dispersed generation system providing system ancillary services through power electronic interfaces. Renew Energy 2011;36(9). ISSN: 0960-1481: 2312e23. http://dx.doi.org/10.1016/j.renene.2011.02.011.

[12] Franco A, Salza P. Strategies for optimal penetration of intermittent renewables in complex energy systems based on techno-operational objectives. Renew Energy 2011;36(2). ISSN: 0960-1481:743e53. http://dx.doi.org/ 10.1016/j.renene.2010.07.022.

[13] Kazempour SJ, Moghaddam MP, Haghifam MR, Yousefi GR. Electric energy storage systems in a market-based economy: comparison of emerging and traditional technologies. Renew Energy 2009;34(12). ISSN: 0960-1481:2630e 9. http://dx.doi.org/10.1016/j.renene.2009.04.027.

[14] Behrangrad M, Moghadam M, Sheikh-El-Eslami M. Long term energy efficiency trading as an approach for the competition improvement in the electricity markets. In: T\&D IEEE/PES, transmission and distribution conference and exposition; 2008.

[15] Karangelos E, Bouffard F. Towards full integration of demand-side resources in joint forward energy/reserve electricity markets. IEEE Trans Power Syst 2012;27(1):280e9.

[16] Chen Y, Li J. Comparison of security constrained economic dispatch formulations to incorporate reliability standards on demand response resources into Midwest ISO co-optimized energy and ancillary service market. Electr Power Syst Res 2011;81(9). ISSN: 0378-7796:1786e95. http://dx.doi.org/10.1016/ j.epsr.2011.04.009.

[17] Faria P, Vale Z, Soares T, Morais H. Energy and reserve provision dispatch considering distributed generation and demand response. In: 3rd IEEE PES international conference and exhibition on innovative Smart grid technologies (ISGT Europe), Berlin, Germany; 2012.

[18] Jazayeri P, Schellenberg A, Rosehart WD, Doudna J, Widergren S, Lawrence D, et al. A survey of load control programs for price and system stability. IEEE Trans Power Syst 2005;20:1504e9.

[19] Wang J, Wang X, Wu Y. Operating reserve model in the power market. IEEE Trans Power Syst February 2005;20(1):223e9.

[20] Heredia F-J, Rider MJ, Corchero C. Optimal bidding strategies for thermal and generic programming units in the day-ahead electricity market. IEEE Trans Power Syst August 2010;25(3):1504e18.

[21] Hart E, Jacobson M. A Monte Carlo approach to generator portfolio planning and carbon emissions assessments of systems with large penetrations of variable renewables. Renew Energy August 2011:36(8):2278e86.

[22] GAMS. GAMS e the solver manuals, Washington D. C; 2001.

[23] Faria P, Vale ZA, Ferreira J. Demsi e a demand response simulator in the context of intensive use of distributed generation. In: IEEE international conference on systems man and cybernetics (SMC); 2010. pp. 2025e32.

[24] FERC. Wholesale competition in regions with organized electric markets. Available from: http://www.ferc.gov/whats-new/comm-meet/2009/121709/ E-7.pdf. [Visited on December 2013].

[25] Baran ME, Wu FF. Network reconfiguration in distribution systems for loss reduction and load balancing. IEEE Trans Power Deliv 1989;4(2):1401e7. 